\title{
Congreso Científico DICYP 2016: una motivación hacia la investigación en la UNAH
}

\author{
Lesbia Jeannette Buitrago Reyes ${ }^{1}$
}

\section{RESUMEN}

Este artículo analiza la información de los expositores y asistentes en el Décimo Congreso de Investigación Científica organizado por la Dirección de Investigación Científica, DICYP en la Universidad Nacional Autónoma de Honduras, UNAH en agosto 2016.

Se destaca la participación de los profesores y estudiantes en los congresos científicos así como la utilización de fondos concursables disponibles en la UNAH por medio de becas de investigación además recoge las opiniones de los participantes sobre los beneficios o no que aportan este tipo de eventos académicos para la visibilización de los resultados de las investigaciones y la mayor o menor participación según la Facultad a la que pertenecen, así como datos de género, edad, último título obtenido y participación por temas prioritarios de la institución. El estudio tiene un enfoque cuantitativo y alcance descriptivo.

En los resultados obtenidos destacaron las conferencias nacionales con 55 eventos y asistencia del $28.6 \%$ del total, seguido de paneles nacionales con 49 eventos y el $24.9 \%$ de asistencia sumando ambos eventos más de la mitad de los eventos realizados (53.5\%). Las Facultades con mayor asistencia fueron las de Humanidades y Artes y Ciencias con $22 \%$ cada una, seguida por Química y Farmacia con $15 \%$, Ciencias Económicas y Ciencias Sociales con $12 \%$ cada una y con menor asistencia Ingeniería, Ciencias Médicas con 5\% y 2\%, y Ciencias Espaciales y Odontología; con $1 \%$ cada una. Por Centro Regional, UNAH-TEC Danlí presentó mayor asistencia con $38 \%$, seguido de CURC con $26 \%$, CURNO con $14 \%$, CURLP y CUROC $7 \%$ respectivamente, CURLA $5 \%$, CURVA $2 \%$ y UNAH-VS $1 \%$.

\footnotetext{
1 Jefa del Departamento Proyectos de Investigación, DICYP, UNAH: investigacionunah.proyectos.Ib@gmail.com
} 
Dos terceras partes de los participantes (77.55\%) manifestaron haber beneficiado de fondos concursables organizadas por la DICYP, exponiendo la diferencia las razones de su no participación.

Los expositores concluyeron como el valor más importante de los congresos de investigación científica la difusión de los trabajos de investigación (66.1\%), el 17.4\% consideraron que estimulan para escribir artículos científicos, los demás señalaron que mejoran los trabajos por medio de los comentarios efectuados en el congreso, mejoran los aspectos teóricos de las investigaciones y permite discutir los aspectos teóricos de las investigaciones y ninguno considero que no tenían importancia.

Palabras clave: Congresos, investigación científica, becas de investigación.

\section{ABSTRACT}

This article analyzes the information of exhibitors and attendees at the Tenth Congress of Scientific Research organized by the Scientific Research Office (DICYP) at the National Autonomous University of Honduras, UNAH in August 2016.

The participation in the scientific congresses is highlighted. The use of competitive available funds through research grants is portrayed through the opinions of the participants on how this type of academic events benefits the visibility of research results. These academic events also show the greater or lesser participation according to the Faculty to which they belong, data of each researcher such as gender, age, last title obtained and participation according to the institution's top priority list.

Key words: Congresses, scientific research, research grants. 


\section{INTRODUCCIÓN}

El Congreso de Investigación Científica de la UNAH es organizado por la Dirección de Investigación Científica y Posgrado (DICYP), es un evento académico de nivel científico, en el que se reúnen anualmente investigadores, profesores, directores de Institutos, unidades de investigación, grupos de investigación, coordinadores regionales de investigación y profesionales expertos en el tema del evento, para presentar y debatir ponencias, dictar conferencias, participar en mesas redondas, mesas de trabajo o en paneles de comentaristas y compartir inquietudes con expertos nacionales e internacionales en las diversas áreas del conocimiento (Dirección de Investigación Científica y Posgrado, 2016).

Pueden participar los investigadores que deseen presentar avances o resultados de sus investigaciones en los ámbitos de la ciencia, la tecnología y la innovación; así como también los que quieran validar proyectos de investigación o debatir temas y problemas del quehacer académico vinculado a la investigación en esos tres ámbitos, y todos los especialistas de dentro y fuera de la UNAH que manifiesten su interés en el tema. El Congreso tiene un tema central al cual contribuyen todas las participaciones desde diferentes paneles de debate combinando con conferencias magistrales, mesas redondas y de trabajo.

Las modalidades de participación de acuerdo a la convocatoria fueron:

- Conferencias internacionales: Expositor traído del exterior a solicitud de la unidad académica o grupo proponente del tema del congreso, para exponer un tema específico, acompañado de un panel de tres comentaristas para aportar, complementar o disentir con las ideas del conferencista intemacional.

- Conferencias nacionales: Especialista de la universidad o que radique en el país -de los centros regionales, de otras universidades o instituciones y de cualquier nacionalidad- contactado directamente por la unidad o grupo proponente, acompañado de un panel de tres comentaristas para argumentar, complementar o disentir con las ideas del conferencista nacional.

- Paneles nacionales: Presentación de una investigación desde diversos aportes y enfoques con un máximo de cinco especialistas. Se realizó además un Panel especial como programa especial por el cumplimiento de 10 años desde la Reforma Universitaria.

- Paneles internacionales: Igual al panel nacional pero los expositores son internacionales. 
- $\quad$ Mesas de trabajo: Presentación de un diseño o avance de investigación por un investigador 0 un equipo de investigación y dos comentaristas.

- Mesas redondas: Un tema central y varios subtemas complementarios desarrollados por los expositores. con un máximo cinco especialistas.

Este estudio tiene como propósito visualizar quienes participaron en este evento académico, los tipos de eventos que se realizaron; la asistencia por evento, Facultad, Centros Regionales, unidades académicas y administrativas y externos a la universidad; la relación de las ponencias con los temas prioritarios de la UNAH y la tipificación de los expositores en el congreso según edad, grado académico; así como el fomento de las investigaciones al identificar de los expositores quienes habían beneficiado o no de las becas de investigación científicas, tecnológicas o de innovación que la UNAH pone a disposición a profesores y estudiantes, determinando además las razones de los que no lo hicieron.

Se espera que sea fuente de información para conocer el perfil de los investigadores que participan en los congresos científicos así como el involucramiento de todas las unidades académicas de la UNAH en este tipo de evento académico que forma parte del proceso de difusión de la investigación científica. Es importante generar estudios e intercambio de ideas al ser considerados los congresos además de las revistas científicas como una proyección de las investigaciones.

\section{METODOLOGÍA}

Este análisis tiene un enfoque cuantitativo y alcance descriptivo. La primera parte presenta la información de los participantes del Décimo Congreso de Investigación Científica en la UNAH, organizado por la Dirección de Investigación Científica y Posgrado (DICYP) en Ciudad Universitaria en Tegucigalpa en agosto de 2016. Para la segunda parte se utilizó una encuesta con preguntas y respuestas precodificadas que se envió virtualmente a todos los expositores, en las diferentes modalidades ya mencionadas.

\section{Análisis estadístico:}

El estudio se realiza utilizando una encuesta electrónica en sitio web, contacto por email, dirigida a los expositores del Décimo Congreso de Investigación Científica de la UNAH organizado por la Dirección de Investigación Científica y Posgrado (DICYP) 
obteniéndose una muestra de 121 expositores de un total de 398 (30.4\% de respuestas).

Para el análisis de los datos previa codificación de estos, se utilizó la hoja de cálculo de Google adicionando algunos análisis con Hoja de cálculo Excel, realizando un análisis descriptivo.

\section{RESULTADOS}

\section{Primera parte:}

\section{a. Tipos de eventos:}

Se realizaron 167 eventos con un total de 9,052 asistentes, representando la mayor cantidad de eventos las conferencias nacionales con 55 eventos y asistencia del $28.6 \%$ del total de personas que llegaron a este congreso; seguido de paneles nacionales con 49 eventos y el $24.9 \%$ de asistencia. Ambos eventos concentraron a más de la mitad de los asistentes (53.5\%), seguido de las mesas de trabajos y conferencias internacionales con $11.1 \%$ y $11.0 \%$ respectivamente, siguiendo las mesas redondas con $6.6 \%$, posters, panel especial con $2.3 \%$ y paneles internacionales con $1.1 \%$ cada uno respectivamente. Los eventos de inauguración, entrega de premios y de clausura presentaron asistencia de 5.8\%, 2.1\% y 4.2\% respectivamente (Ver cuadro 1).

\section{b. Asistencia}

En el Gráfico 1 puede observarse la mayor asistencia de estudiantes que profesores en todos los eventos, representando mayor diferencia en la conferencia nacional y en el panel nacional, con diferencias de 1,311 y 927 respectivamente; seguido por la conferencia internacional, mesa de trabajo y mesa redonda con diferencias de 319 , 438 y 242 respectivamente permaneciendo la misma tendencia en cifras absolutas. Para tener una percepción relativa de las mismas debería calcularse tasas de participación, pero las mismas no son exactas actualmente. 
Cuadro 1. Décimo Congreso de Investigación Científica UNAH, 2016 por cantidad y asistencia a eventos

\begin{tabular}{|l|c|c|c|}
\hline \multicolumn{1}{|c|}{ Eventos realizados } & $\begin{array}{c}\text { Cantidad } \\
\text { de eventos }\end{array}$ & $\begin{array}{c}\text { Asistencia } \\
\text { por evento }\end{array}$ & $\%$ \\
\hline Evento de inauguración & 1 & 525 & $5.8 \%$ \\
\hline Conferencia internacional (Cl) & 12 & 992 & $11.0 \%$ \\
\hline Conferencia nacional (CN) & 55 & 2588 & $28.6 \%$ \\
\hline Panel internacional (PI) & 1 & 97 & $1.1 \%$ \\
\hline Panel nacional (PN) & 49 & 2255 & $24.9 \%$ \\
\hline Mesa de trabajo (MT) & 27 & 1009 & $11.1 \%$ \\
\hline Mesa de redonda (MR) & 14 & 595 & $6.6 \%$ \\
\hline Póster (P) & 5 & 212 & $2.3 \%$ \\
\hline Entrega de premios & 1 & 189 & $2.1 \%$ \\
\hline Panel especial & 1 & 211 & $2.3 \%$ \\
\hline Evento de clausura & 1 & 379 & $4.2 \%$ \\
\hline Total & 167 & 9052 & $100.0 \%$ \\
\hline
\end{tabular}

Fuente: Dirección de Investigación Científica, 2016.

Gráfico 1. Décimo Congreso de investigación Científica UNAH, 2016 por asistencia a tipos de evento

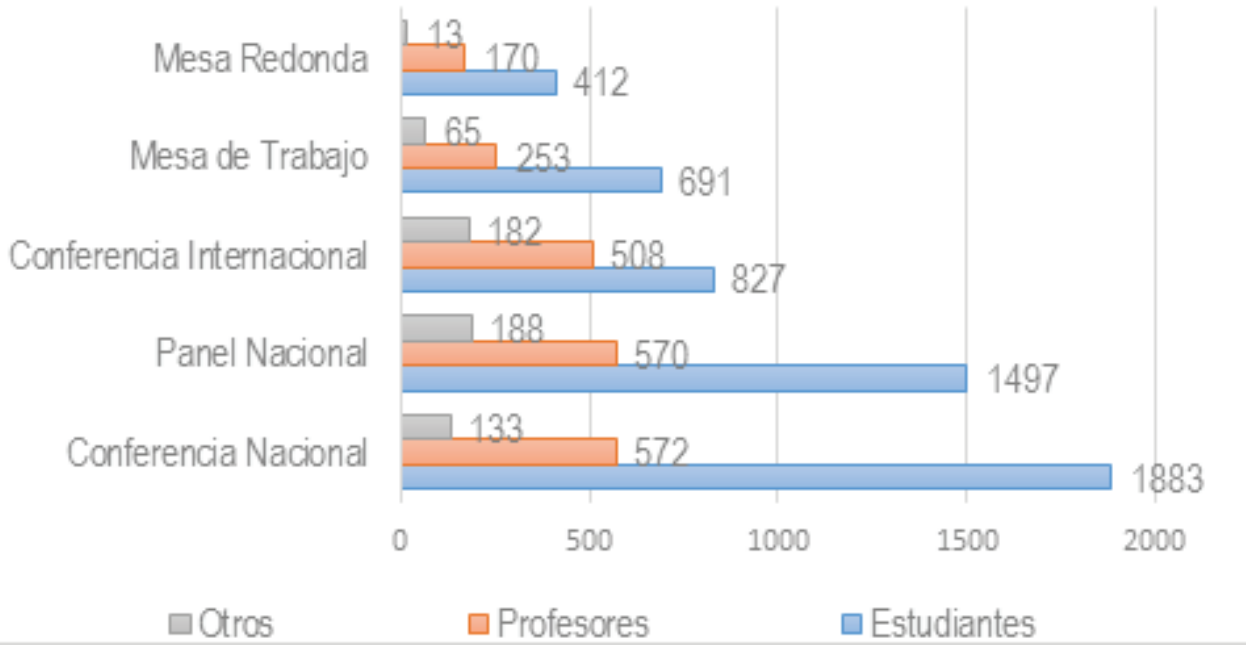

Fuente: Dirección de Investigación Científica, 2016. 
En el Gráfico 2 puede observarse que las Facultades con mayor asistencia fueron las de Humanidades y Artes y Ciencias con $22 \%$ cada una, seguida por Química y Farmacia con $15 \%$, Ciencias Económicas y Ciencias Sociales con $12 \%$ cada una y con menor asistencia Ingeniería, Ciencias Médicas con 5\% y 2\%, y Ciencias Espaciales y Odontología; con $1 \%$ cada una.

\section{Gráfico 2. Décimo Congreso de Investigación Científica UNAH, 2016 asistencia por facultad}

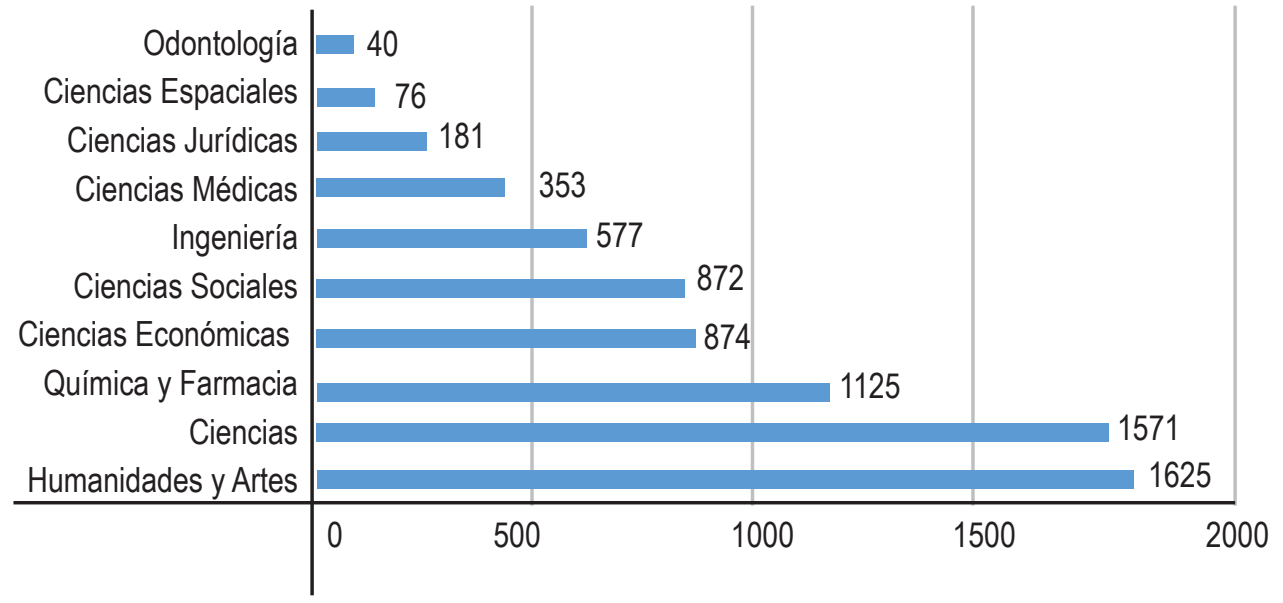

Fuente: Dirección de Investigación Científica, 2016.

\section{Gráfico 3. Décimo Congreso de Investigación Científica UNAH, 2016 asistencia por centro regional}

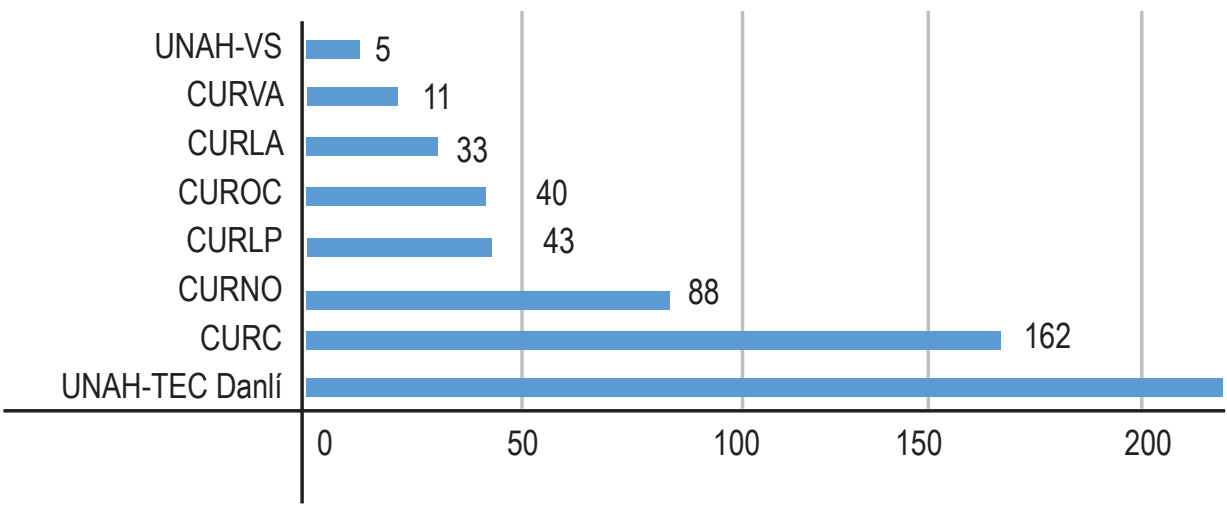

Fuente: Dirección de Investigación Científica, 2016. 
Por Centro Regional, UNAH-TEC Danlí presentó mayor asistencia con 38\%, seguido de CURC con $26 \%$, CURNO con $14 \%$, CURLP y CUROC $7 \%$ respectivamente, CURLA $5 \%$, CURVA $2 \%$ y UNAH-VS 1\% (ver gráfico 3). La asistencia de otras unidades académicas y administrativas de la UNAH así como instancias externas fue de 1,145 participantes, siendo de unidades académicas 588 y de externos a la UNAH de 557.

\section{c. Temas prioritarios}

La UNAH dispone de 13 temas prioritarios aglutinados en cuatro ejes de investigación, que son: Eje de investigación $\mathrm{N}^{\circ} 1$ : Desarrollo económico y social, el cual incluye 3 temas prioritarios: 1. Infraestructura y desarrollo territorial; 2. Pobreza e inequidad y 3. Globalización, productividad y competitividad. El Eje de investigación N 2: Democracia y Gobernabilidad incluye el tema prioritario 4. Reforma del Estado, 5. Democracia, ciudadanía y participación y 6.Conflictividad política y social; Eje de investigación $\mathrm{N}^{\circ}$ 3: Población y condiciones de vida con los temas prioritarios 7.Población, necesidades básicas y transición demográfica, 8.Ciencia, cultura y educación, 9.Salud, Estado y Sociedad, 10.Seguridad y violencia y Eje de investigación N ${ }^{\circ} 4$ : Ambiente, biodiversidad y desarrollo con los temas prioritario 11. Cambio climático y vulnerabilidad, 12. Seguridad alimentaria y nutricional, y 13. Desarrollo energético: fuentes, impacto y política.

Las ponencias presentadas en este congreso fueron mayoritariamente del tema prioritario número 8 , seguido del tema 3 y 9 , ocuparon mismo lugar los temas 7 y 11 , seguido de los temas 13, 12 y 1 , finalmente se tienen los temas 4,10 y 6 . Puede observarse la ausencia de varios temas prioritarios, como son los $\mathrm{N}^{\circ} 2$ Democracia y gobernabilidad, $N^{\circ} 5$ Democracia, ciudadanía y participación y el tema $N^{\circ} 6$ Conflictividad política y social, todos ellos están dentro del Eje de investigación $\mathrm{N}^{\circ} 2$. Destaca la importancia de desarrollar investigaciones en ellos pues están llamados a ser atendidos institucionalmente (ver Gráfico 4).

\section{d. Expositores según grado académico}

El Cuadro 3 presenta el último grado académico de los expositores por Facultad, Centro Regional y otras Unidades Académicas. Puede observarse que casi la mitad (49\%) tienen grado de maestría, seguido por Licenciatura (31\%). Con doctorado se presentaron $15 \%$, teniendo menos representatividad quienes se clasificaron teniendo Especialidad, Subespecialidad y estudiante universitario con $3 \%, 2 \%$ y $0.3 \%$ respectivamente. 


\section{Gráfico 4. Décimo Congreso de Investigación Científica UNAH, 2016 número de ponencias por temas prioritarios}

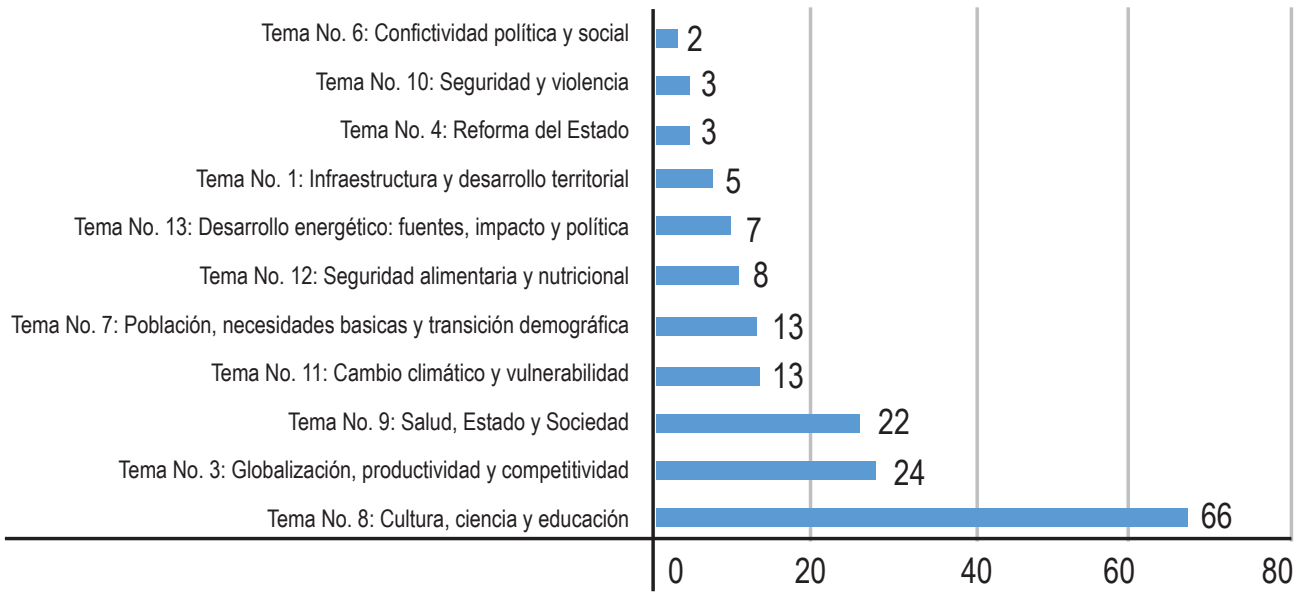

Fuente: Dirección de Investigación Científica, 2016

\section{Cuadro 5. Décimo Congreso de Investigación Científica UNAH, 2016 expositores por último grado académico}

\begin{tabular}{|c|c|c|c|c|c|c|c|}
\hline $\begin{array}{l}\text { Unidad } \\
\text { académica }\end{array}$ & Doctorado & Maestría & Especialidad & $\begin{array}{l}\text { Sub- } \\
\text { especialidad }\end{array}$ & Licenciatura & $\begin{array}{l}\text { Estudiante } \\
\text { universitario }\end{array}$ & Total \\
\hline Facultad & 33 & 127 & 4 & 1 & 67 & 1 & 233 \\
\hline Ciencias & 7 & 22 & & & 16 & 1 & 47 \\
\hline Ciencias Económicas & 6 & 21 & & & 13 & & 40 \\
\hline Ciencias Espaciales & 2 & 17 & & & 6 & & 25 \\
\hline Ciencias Jurídicas & 1 & 4 & & & 2 & & 7 \\
\hline Ciencias Médicas & 1 & 9 & 2 & & 1 & & 13 \\
\hline Ciencias Sociales & 11 & 19 & 1 & & 13 & & 44 \\
\hline Humanidades y Artes & 3 & 25 & & 1 & 8 & & 37 \\
\hline Ingeniería & & 3 & & & 1 & & 4 \\
\hline Química y Farmacia & 2 & 7 & & & 7 & & 16 \\
\hline Centros regional & 3 & 26 & 3 & & 22 & & 5 \\
\hline CURC & & 4 & & & 3 & & 7 \\
\hline CURLA & & 3 & 1 & & 3 & & 7 \\
\hline CURLP & 1 & 2 & & & 1 & & 4 \\
\hline CURNO & & 3 & & & 4 & & 7 \\
\hline CUROC & & 8 & 1 & & 1 & & 10 \\
\hline UNAH-TEC Danli & 2 & 6 & 1 & & 8 & & 17 \\
\hline UNAH-VS & & & & & 2 & & 2 \\
\hline Otros & 23 & 41 & 3 & & 36 & 8 & 111 \\
\hline Total general & 59 & 194 & 10 & 1 & 125 & 9 & 398 \\
\hline
\end{tabular}

Fuente: Dirección de Investigación Científica, 2016 


\section{Segunda parte:}

La información para esta segunda parte fue obtenida por medio de una encuesta dirigida a los expositores en el congreso. Los participantes fueron mayoritariamente de sexo masculino $(61.7 \%)$, teniendo mayor participación el grupo de edad entre los 30-39 años con el 30\%, seguido del grupo 40-49 años con el 28\%, el grupo 20-29 con el 19\%, el grupo de 50-59 con el 17\% y el grupo con menor participación es el de 60 y más con el $6 \%$. Esa estructura posiblemente está influida mayoritariamente por la estructura por edad del cuerpo de profesores de la UNAH.

\section{Gráfico 5. Expositores Décimo Congreso de Investigación Científica por edad, 2016}

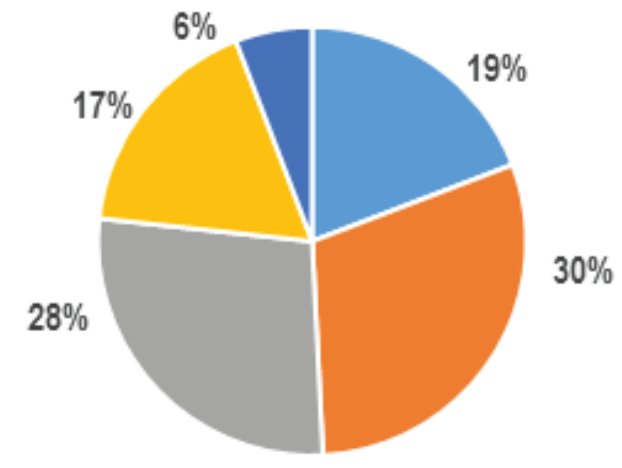

$-20-29=30-39=40-49=50-59-60$ y más

Fuente: Encuesta Décimo Congreso de investigación Científica, UNAH, 2016

En cuanto a la situación laboral del total de participantes, los profesores representaron $90.2 \%$ y los estudiantes $9.8 \%$. El $81 \%$ del total de expositores respondió haber participado en congresos anteriormente y $19 \%$ indicaron que era la primera vez que participaban en este tipo de evento.

\section{Apoyo de las becas de investigación}

Las becas de investigación científica, tecnológica o de innovación son asignaciones otorgadas a profesores-investigadores y/o estudiantes de grado y posgrado para el desarrollo de proyectos de investigación. Tienen como objetivos motivar a los profesores y estudiantes de la UNAH a que se integren a los procesos de investigación sobre ciencia, tecnología e innovación y sobre la realidad universitaria y nacional; fortalecer las iniciativas en el campo de la investigación; desarrollar las prioridades de 
investigación científica que se ha propuesto la universidad y estimular a los estudiantes para desarrollar proyectos de investigación científica, tecnológica o de innovación. (Dirección de Investigación Científica y posgrado, DICYP, UNAH, 2015).

Dos terceras partes de los participantes (77.55\%) manifestaron haber beneficiado de fondos concursables organizadas por la DICYP. Las respuestas del grupo que respondió afirmativamente pueden observarse en el gráfico 4: 69\% había realizado su investigación por medio de una beca básica ${ }^{2}$, seguido por becas sustantivas ${ }^{3}$ (19\%), mientras que $8 \%$ estudiantes de grado 4 que obtuvieron beca, el $4 \%$ fueron estudiantes de posgrado y nadie reportó haber obtenido beca de docente egresado de posgrado 5 .

\section{Gráfico 6. Expositores Décimo Congreso de Investigación Científica por tipo de beca de investigación, 2016}

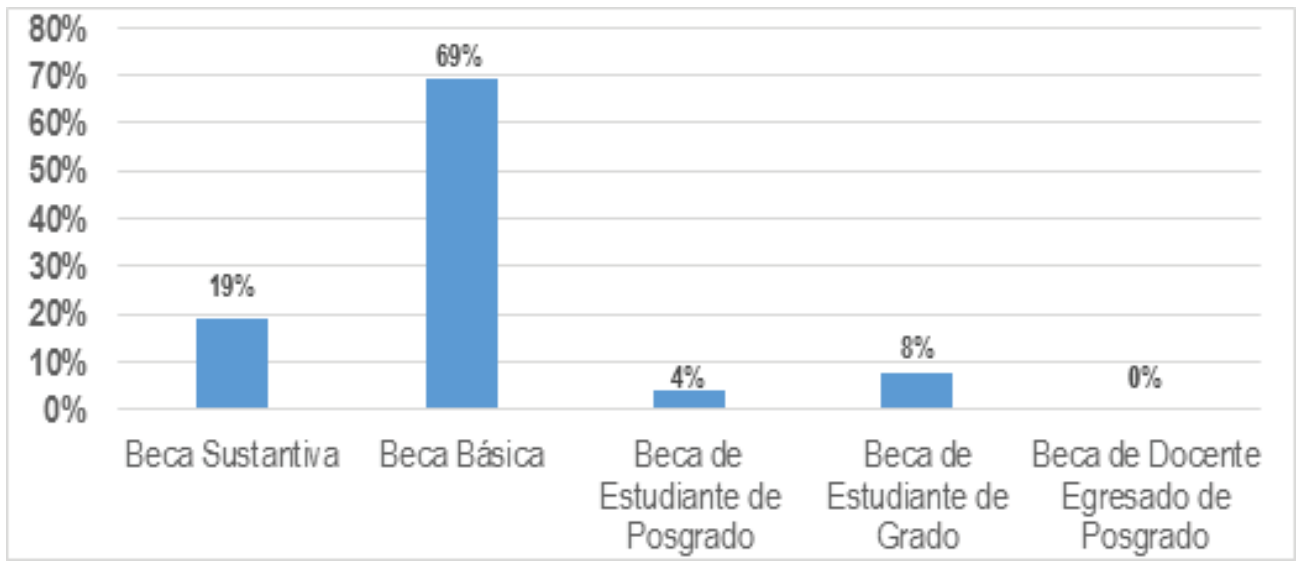

Fuente: Encuesta Décimo Congreso de investigación Científica, UNAH, 2016

Del $77.5 \%$ de participantes que realizaron su investigación con una beca de investigación $65.6 \%$ la había recibido una vez, $25 \%$ hasta dos veces y el $9.4 \%$ indicó que había beneficiado tres veces o más.

${ }_{2}$ Asignaciones otorgadas a profesores-investigadores para el desarrollo de proyectos de investigación.

${ }^{3}$ Asignaciones otorgadas para el desarrollo de proyectos inter o multidisciplinarios de investigación por un mínimo de dos profesores investigadores activos.

${ }^{4}$ Asignaciones otorgadas a estudiantes de grado y posgrado de la UNAH con excelencia académica, presentan un proyecto de investigación de alta calidad y pertinencia, avalado por un profesor universitario y que responde a las prioridades de investigación de la universidad y de la unidad académica a la que se encuentra adscrito.

${ }^{5}$ Asignaciones otorgadas a los profesores de la UNAH para la finalización de sus tesis o trabajos de graduación de posgrado, que presenten por lo menos un $60 \%$ de avance de su tesis. 
Del $22.5 \%$ que no participó con una investigación producto de una beca de investigación se le consultó el motivo por el cual no había recibido una beca de investigación con fondos de la DICYP, expresando el $13.4 \%$ que la habían solicitado y no había sido aprobada, $13.4 \%$ dijo que prefería hacerlo con sus propios fondos, $11 \%$ no cumplía requisitos para optar a una beca de investigación y la mayoría (62.2\%) se refirió a otros, de los cuales la mitad plantearon que aunque no lo habían solicitado pensaban hacerlo próximamente, el $18.8 \%$ no cumplía requisitos, y el resto fue por desconocimiento y porque el monto de las becas de investigación es muy bajo.

Dos tercios de los participantes consideraron como el valor más importante de los congresos de investigación científica la difusión de los trabajos de investigación (66.1\%), el $17.4 \%$ consideraron que estimulan para escribir artículos científicos, $6.6 \%$ señalaron que mejoran los trabajos por medio de los comentarios efectuados en el congreso, $5 \%$ indicaron que permiten mejorar los aspectos teóricos de las investigaciones, el $4.1 \%$ permite discutir los aspectos teóricos de las investigaciones. Sólo $0.8 \%$ lo valoraron como para reconocer méritos y ninguno considero que no tenían importancia. Esto coincide con lo expresado por García Canal (2003) citado por (Ruiz-Santos \& Meroño-Cerdán, 2007), que considera que la presentación de trabajos en conferencias: 1) puede aumentar sustancialmente las posibilidades de publicar en una buena revista científica, pues el trabajo se puede beneficiar de comentarios que contribuyan a su mejora, al tiempo que los autores pueden hacerse notar ante los editores de la revista; 2) permite a los autores acreditar la calidad de la investigación en curso, pues buena parte de las conferencias tienen un riguroso proceso de filtrado y evaluación de los trabajos presentados y 3) se amplía la red personal de los investigadores, estableciendo contactos con colegas de la misma especialidad que trabajan en otras universidades.

\section{CONCLUSIONES}

La investigación científica es una de las funciones básicas de las universidades, la UNAH la ha impulsado estableciéndola como una política institucional con apoyos financieros y de capacitación. Los congresos científicos marcan su importancia al reflejar a estudiantes y profesores que hacen investigación así como a los asistentes como buscadores de nuevos conocimientos.

El Décimo Congreso de Investigación Científica organizado por la DICYP muestra la mayor asistencia de estudiantes que profesores en todos los eventos, representando 
la mayor cantidad de eventos las conferencias nacionales con 55 eventos y asistencia del $28.6 \%$ del total, seguido de paneles nacionales con 49 eventos y el $24.9 \%$ de asistencia sumando ambos eventos más de la mitad de los eventos realizados (53.5\%).

Las ponencias presentadas en este congreso fueron mayoritariamente del tema prioritario número 8: Ciencia, cultura y educación, seguido del tema 3: Globalización, productividad y competitividad y 9: Salud, Estado y Sociedad, los cuales se vinculan a las facultades con mayor participación que fueron las de Humanidades y Artes y Ciencias con $22 \%$ cada una, seguida por Química y Farmacia con $15 \%$, Ciencias Económicas y Ciencias Sociales con $12 \%$ cada una, presentando los Centros Regionales como UNAH-TEC Danlí la mayor asistencia con 38\%, seguido de CURC con $26 \%$ y CURNO con $14 \%$.

Dos terceras partes de los participantes (77.6\%) manifestaron haber beneficiado de fondos concursables organizadas por la DICYP. Del grupo que respondió afirmativamente, $69 \%$ había realizado su investigación por medio de una beca básica, seguido por becas sustantivas (19\%), mientras que $8 \%$ de estudiantes de grado que obtuvieron beca, el $4 \%$ fueron estudiantes de posgrado y nadie reportó haber obtenido beca de docente egresado de posgrado.

Los expositores consideraron el valor más importante de los congresos de investigación científica la difusión de los trabajos de investigación (66.1\%), el 17.4\% consideraron que estimulan para escribir artículos científicos, los demás señalaron que mejoran los trabajos por medio de los comentarios efectuados en el congreso, mejoran los aspectos teóricos de las investigaciones y permite discutir los aspectos teóricos de las investigaciones y ninguno considero que no tenían importancia.

Se destaca la importancia de los congresos científicos como plataforma para aquellos investigadores que presentan sus resultados de investigación con el objetivo de compartir con sus pares un debate científico, tecnológico o de innovación que permite generar y difundir el conocimiento.

\section{AGRADECIMIENTOS}

A la Dirección de Investigación Científica y Posgrado (DICYP) por las fuentes de información disponibles para la realización de este estudio. 


\section{BIBLIOGRAFÍA}

Dirección de Investigación Científica y Posgrado (2016). Dirección de Investigación Científica y Posgrado, UNAH. Recuperado de: https://dicyp.unah.edu.hn/ Dirección de Investigación Científica y posgrado, DICYP, UNAH. (2015). Compendios de Investigación Científica, serie 3. Tegucigalpa: UNAH.

Ruiz-Santos, C. \& Meroño-Cerdán, A. (2007). Utilidad de los congresos científicos en la difusión del conocimiento: percepción del investigador español en Economía de la Empresa. Recuperado de: http://www.cyta.com.ar/ta0602/v6n2a1.htm 